\title{
Monitoring Organic Reactions by Micellar Electrokinetic Chromatography
}

\author{
Chun Yang, ${ }^{1,2}$ Chunyang Chang, ${ }^{1}$ Jun Wang, ${ }^{1}$ Qishu Qu, ${ }^{1}$ Xiaoya Hu, ${ }^{1}$ and Yang Wang1 \\ ${ }^{1}$ College of Chemistry \& Chemical Engineering, Yangzhou University, Yangzhou 225002, China \\ ${ }^{2}$ Key Laboratory of Separation Sciences for Analytical Chemistry, Dalian Institute of Chemical Physics, CAS, Dalian 116023, China
}

Correspondence should be addressed to Chun Yang, cyang01@126.com

Received 19 December 2011; Accepted 29 January 2012

Academic Editors: E. Boselli, M. C. Monti, and A. I. Suarez

Copyright (C) 2012 Chun Yang et al. This is an open access article distributed under the Creative Commons Attribution License, which permits unrestricted use, distribution, and reproduction in any medium, provided the original work is properly cited.

\begin{abstract}
A method was established to monitor organic reactions by micellar capillary electrokinetic chromatography (MEKC). After optimizing conditions such as the composition of the solvents, the surfactant, and the apparent $\mathrm{pH}\left(\mathrm{pH}^{*}\right)$ of the system, the method was utilized to analyze the reaction of glycidyl methacrylate (GMA) and allyl amine. The main products were identified in the electropherograms. The reaction procedure was monitored in real time. This method was found to have common applicability, being able to separate and detect nonaqueous soluble, nonionic, and low-UV-Vis absorbance compounds. It provides a rapid and low-cost way to understand organic reactions and to direct synthesis works.
\end{abstract}

\section{Introduction}

Capillary electrophoresis (CE) is a rapid and high-performance method of separation and analysis [1]. It possesses advantages such as high efficiency, high speed, ease to be automated, low sample consumption, low cost, and multimode availability. After decades of development, CE plays important roles in the fields of chemistry, biology, pharmaceutical, environment, toxicology, medicine, and forensic chemistry [2-10].

It is very important to analyze the products of an organic reaction, as well as to monitor the related kinetics or reaction procedure. The related methods are mainly focused on elemental analysis, nuclear magnetic resonance (NMR), mass spectrometry (MS), infrared radiation (IR), and ultraviolet visible (UV-Vis) spectrometry. Many of these methods are usually obstructed by the limited purification of the products from complex reactions.

A chromatographic method (gas or liquid) can be used to simultaneously separate and detect complex samples. It enables the analysis and monitoring of organic reactions in real time [11]. CE is a method which can analyze charged analytes in aqueous solution. CE could also be an important method to analyze chemical reactions, playing a role in the research of reaction kinetics $[5,12]$, asymmetric transformation of amino acids [13, 14], catalytic oxidation [15], assembling of nanoparticles [16], protein interaction [17], and polymerase chain reaction (PCR) [18-20]. One of the modes of CE, micellar electrokinetic chromatography (MEKC), introduced surfactants as pseudostationary phases. In such a CE operation, analytes are not only separated in accordance with their mobility but also based on their distributions in the pseudostationary phases. As a result, neutral compounds without any charge could also be separated in that case when they appear in different concentrations in the micelle.

In this paper, we give an example to explore the possibility to establish a universal method to study organic reactions by MEKC. Most of the compounds involved in an organic reaction, neutral or charged, water soluble, or insoluble, could be found in the electropherograms. The changing of relative peak areas of both the reactants and the products was used to monitor the reaction process.

\section{Experimental Section}

2.1. Chemicals. Allyl amine was purchased from Haopeng Chemical Plant (Jinan, Shandong, China). GMA was purchased from Sigma. Cetyltrimethyl ammonium bromide 
(CTAB) was purchased from Lingfeng Chemical Reagents Co., Ltd (Shanghai, China). Imidazole was purchased from Sinopharm Chemical Reagents Co., Ltd (Shanghai, China). Other reagents were of analytical grade.

2.2. Sample Preparation. GMA and allyl amine (either $10 \mu \mathrm{L}$ ) were dissolved in $2 \mathrm{~mL}$ solvent (methanol/acetonitrile with $\mathrm{v} / \mathrm{v}$ ratio $65: 35)$. Before injection, the mixture was kept in a water bath at $60^{\circ} \mathrm{C}$ for a certain period of time.

2.3. Micellar Electrokinetic Chromatography. Capillary electrophoresis was carried out on a TriSepTM-2100 system (Unimicro Anal Tech Co, Shanghai, China). The capillary (100 um i.d., $360 \mu \mathrm{m}$ o.d.) was purchased from Reafine Chromatographic Device Co., Ltd. (Yongnian, Hebei, China). The running buffer was filtered through $0.45 \mu \mathrm{m}$ (PTFE) and degassed ultrasonically before use.

\section{Results and Discussion}

3.1. General Characters of the Reaction. The reaction of GMA and allyl amine was originally considered for the preparation of a multifunctionality monomer with high reactivity like a methacrylate. As showed in Figure 1, the reaction leads to more than 4 products. Products I and II are two target compounds with both an amino and a methacryl group. They are made from the amination of the epoxy group in GMA. However, multifunctional compounds could carry through reactions via different parts of the molecules, resulting in more than one product. Similarly organic reactions always lead to various by-products as well as the targeted product. Thus, it is necessary to thoroughly analyze and understand the reactions in many ways before expanding their applications. The peaks of the products in the electropherograms are identified mainly according to their mobilities, that is, small basic compounds migrate faster than big neutral or acidic ones.

3.2. Effect of $p H^{*}$. The $\mathrm{pH}^{*}$ of the solution can obviously change the electroosmotic flow, as well as the charged status of the ionic analytes. The two main products of the reaction are basic compounds. Phosphorous acid $(1 \mathrm{~mol} / \mathrm{L})$ was used to adjust the $\mathrm{pH}^{*}$ of the running buffer. To overall compromise the resolution, resolved peak number, and total separation time, the $\mathrm{pH}^{*}$ of the buffer was fixed at 6.50. Figure 2 is shown as an example of various electropherograms resulted from different $\mathrm{pH}^{*} \mathrm{~s}$.

3.3. Solvent Composition and Ratio. One of the reactants, allyl amine, is water soluble and the other, GMA. is insoluble in water. As to the products, their solubility could vary in a wide range. To dissolve all the reactants and products, solvents like methanol, acetonitrile, and their mixtures were tried. Finally, the mixture of methanol/acetonitrile $(65: 35 \mathrm{v} / \mathrm{v})$ was used to run the electrophoresis. In this case, it became a nonaqueous mode of CE $[4,21]$.

3.4. Concentration of CTAB. CTAB was used to form a micelle in which neutral compounds can be separated. On
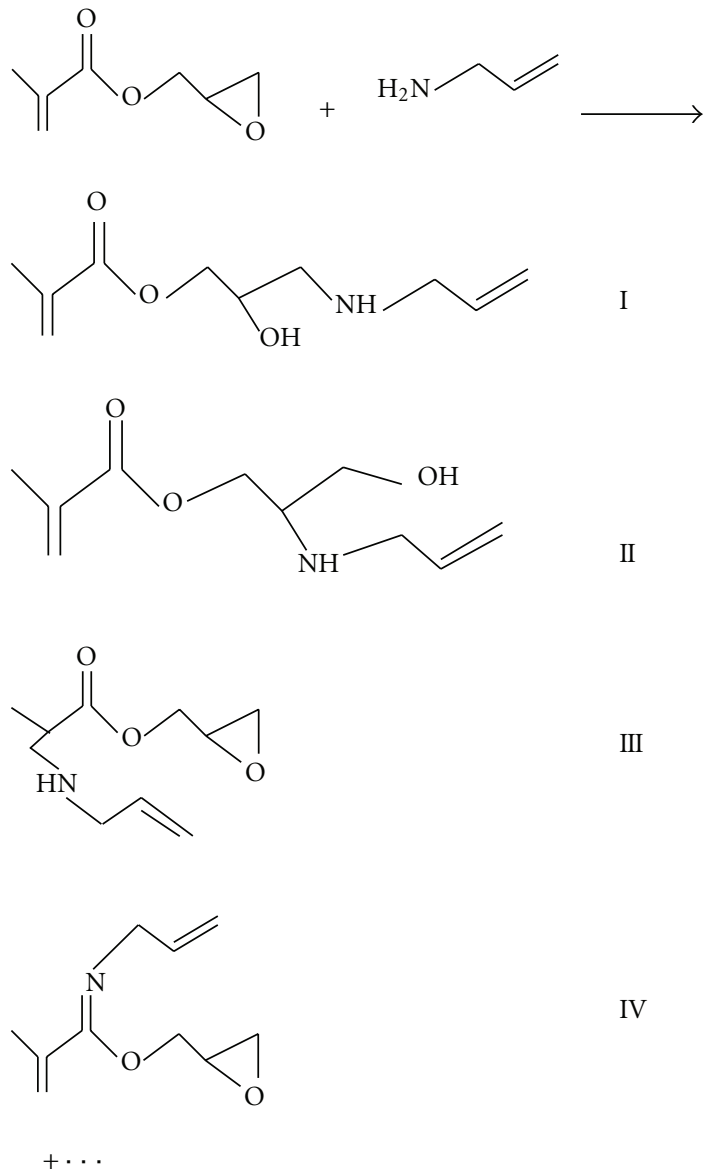

IV

FIGURE 1: The reaction products of glycidyl methacrylate and allyl amine.

the other hand, CTAB can affect the electroosmotic flow (EOF) in CE, by absorption on the inner wall of the capillary and neutralizing the deprotonated silanol groups. Hence, increasing the concentration of CTAB could decrease the EOF and prolong the total separation time. Finally, CTAB was used in the concentration of $35 \mathrm{mmol} / \mathrm{L}$.

3.5. Reaction Process of GMA and Allyl Amine Monitored by $M E K C$. The electropherograms indicating the reaction process are showed in Figure 3. The peaks of GMA and allyl amine appear at about $13 \mathrm{~min}$, and $3 \mathrm{~min}$ respectively. Thiourea (16 min in Figure 3 ) was added as an internal standard substance. As the reaction progresses, the heights of the two reactants declined gradually. About $30 \mathrm{~min}$ after the blend of the reactants, the peak of the first product (product I in Figure 1) appears (6 min in Figure 3(a)). The appearance of the peak of the second product (compound II in Figure 1) was observed $90 \mathrm{~min}$ after the blending (about $3.7 \mathrm{~min}$ in Figure 2(b)). $150 \mathrm{~min}$ after the reaction initiation, the first by-product, compound III in Figure 1, appears in the electropherogram (10.2 min in Figure 3(c)). Finally, allyl amine was completely consumed (about 12 hours later), then the seventh peak appears in the electropherogram (11.1 min in Figure 3(d)). Compounds III and IV were formed by 


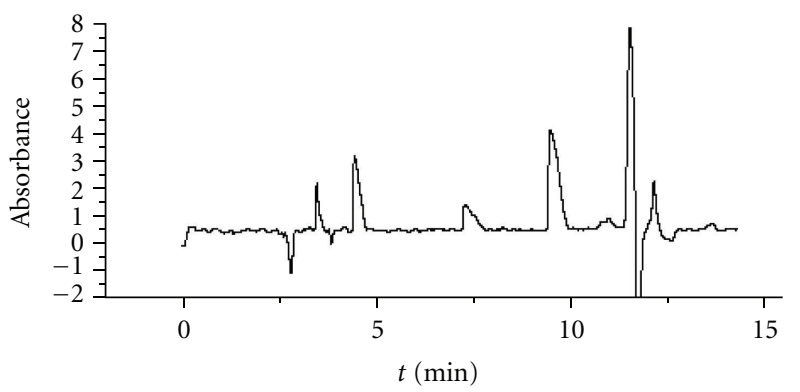

(a)

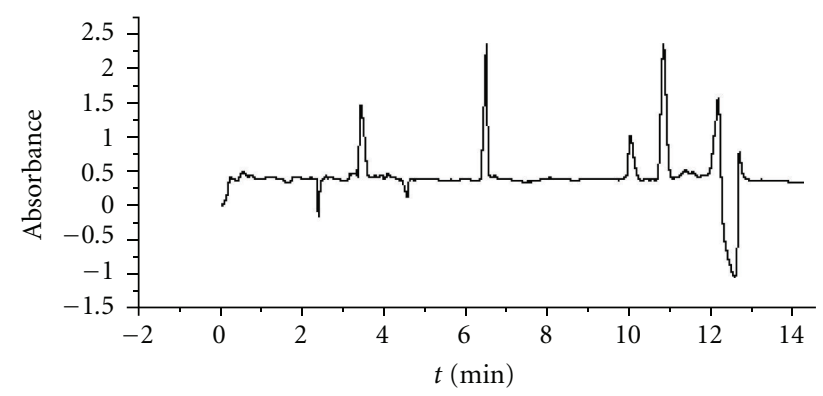

(b)

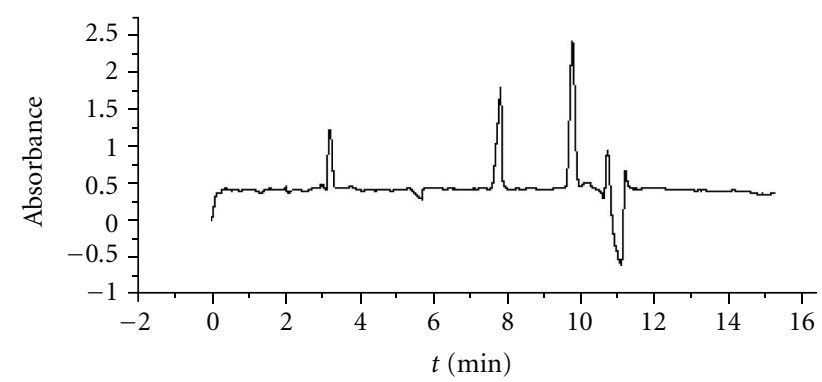

(c)

Figure 2: The effect of $\mathrm{pH}^{*} \mathrm{~s}$ on the separation of the reaction mixture. Buffer: Imidazole $15 \mathrm{mM}$, CTAB $35 \mathrm{mM}$ in methanol/acetonitrile 65/35 (v/v). $\mathrm{pH}^{*}$ (adjusted with $\mathrm{H}_{3} \mathrm{PO}_{3}$ ): (a) 6.01, (b) 6.50, and (c) 7.02. Voltage: $20 \mathrm{kV}$. Detection wavelength: $214 \mathrm{~nm}$. Injection: $5 \mathrm{kV} / 5 \mathrm{sec}$. Capillary: $50 \mu \mathrm{m}$ I.D., $40.0 \mathrm{~cm}$ total $29 \mathrm{~cm}$ effective.

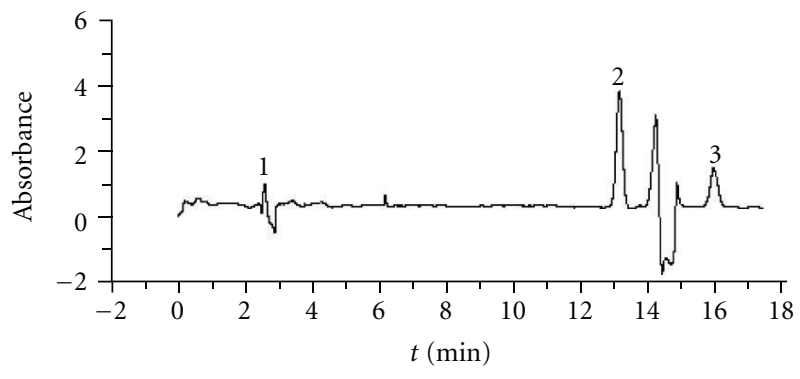

(a)

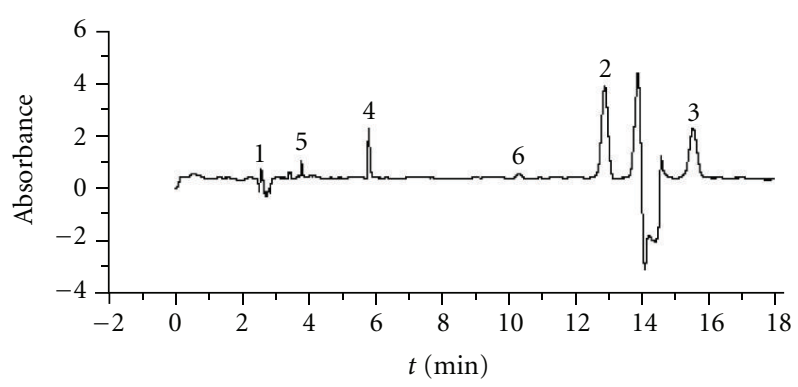

(c)

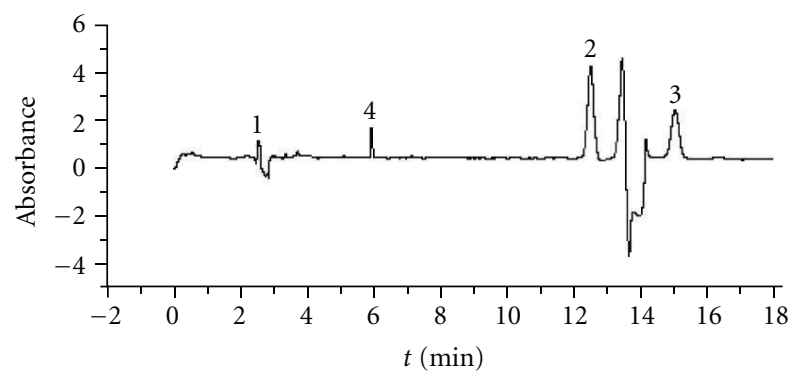

(b)

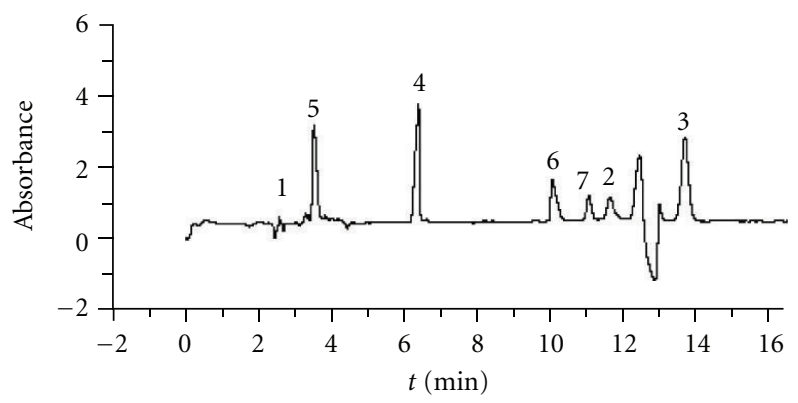

(d)

Figure 3: Reaction process of GMA and allyl amine. Time of reaction: (a) 30 min, (b) 90 min, (c) 150 min, and (d) 12 h. Peaks: (1) allyl amine, (2) GMA, (3) thiourea, (4) 2-hydroxyl-3-allylamino-propyl methacrylamide (product I in Figure 1), (5) 2-allylamino-3-hydroxylpropyl methacrylamide (product II in Figure 1), (5) glycidyl ( $\alpha$-allyl amino-methacrylate) (product III in Figure 1), and (6) product IV in Figure 1. Electrophoresis conditions are the same as in Figure 2. 


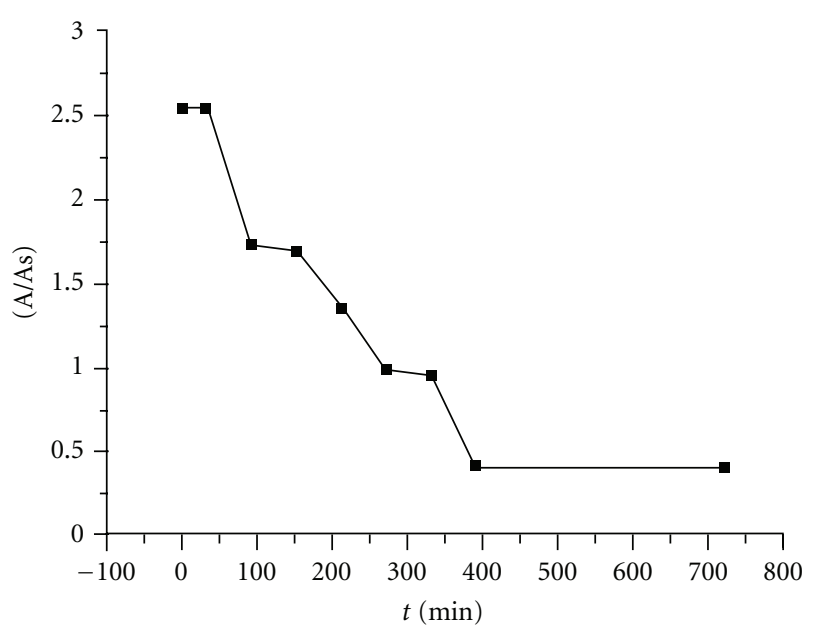

(a)

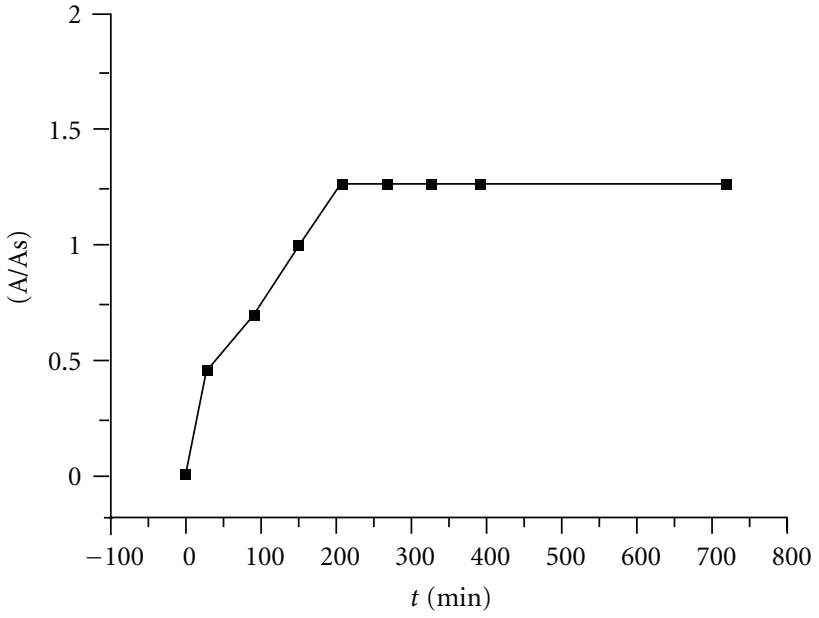

(b)

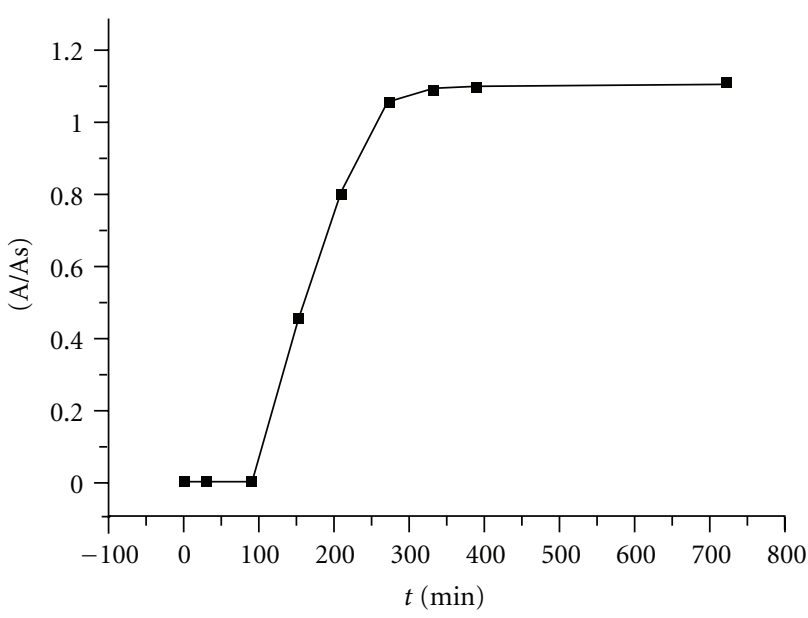

(c)

FIGURE 4: The peak area ratios (to that of thiourea) varied versus time. (a) GMA, (b) compound I, and (c) compound II. Electrophoresis conditions are the same as in Figure 2.

the consumption of methacryl group of GMA and here were regarded as by-products in this reaction.

The reaction can be elucidated from the variation of the area ratios of the compounds (to that of thiourea). Figure 3 illustrates that GMA was the consumed in different ways, that is, it was consumed by the production of various products. It was used to mainly produce compounds I and II during 0-150 min and 150-350 min. This could be confirmed from the peak area ratio variations of compounds I and II, which are maximized at $200 \mathrm{~min}$, and $300 \mathrm{~min}$ respectively (Figures $4(\mathrm{~b})$ and 4(c)). The additional reaction time resulted in the production of by-products, like compounds II and IV.

\section{Concluding Remarks}

A useful method based on nonaqueous MEKC was developed to analyze organic reactions. An example was given to identify the various products of the reaction of GMA and allyl amine and to monitor the reaction process. It was found both neutral and charged, low UV-Vis absorbance compounds could be separated and identified with such an applicative method. With the popularization of CE in analytical fields, this method enables us to analyze and monitor organic reactions in an on-line, real-time, and lowcost method.

\section{Acknowledgments}

This work was financially supported by SRF (Ministry of Education, China) and Key Laboratory of Separation Sciences for Analytical Chemistry (KL1005, Dalian Institute of Chemical Physics, Chinese Academy of Sciences).

\section{References}

[1] S. Hjertén, "High-performance electrophoresis: the electrophoretic counterpart of high-performance liquid chromatography," Journal of Chromatography A, vol. 270, pp. 1-6, 1983. 
[2] J. H. Xiong, W. B. Zhang, G. W. Xu, and Y. K. Zhang, "Advances in nonaqueous capillary electrophoresis," Chinese Journal of Chromatography, vol. 18, no. 3, pp. 218-223, 2000.

[3] J. Stocks and N. E. Miller, "Capillary electrophoresis to monitor the oxidative modification of low density lipoproteins," Journal of Lipid Research, vol. 39, no. 6, pp. 1305-1309, 1998.

[4] P. F. G. De Sa, J. M. Treubig Jr., P. R. Brown, and J. A. Dain, "The use of capillary electrophoresis to monitor Maillard reaction products (MRP) by glyceraldehyde and the epsilon amino group of lysine," Food Chemistry, vol. 72, no. 3, pp. 379384, 2001.

[5] M. Vaher, S. Ehala, and M. Kaljurand, "On-column capillary electrophoretic monitoring of rapid reaction kinetics for determination of the antioxidative potential of various bioactive phenols," Electrophoresis, vol. 26, no. 4-5, pp. 990-1000, 2005.

[6] X. Sun, W. Jin, D. Li, and Z. Bai, "Measurement of alkaline phosphatase isoenzymes in individual mouse bone marrow fibroblast cells based on capillary electrophoresis with on-capillary enzyme-catalyzed reaction and electrochemical detection," Electrophoresis, vol. 25, no. 12, pp. 1860-1866, 2004.

[7] J. Pédron, R. Maldiney, M. Brault, and E. Miginiac, "Monitoring of hapten-protein coupling reactions by capillary zone electrophoresis: improvement of abscisic acid-bovine serum albumin coupling and determination of molar coupling ratios," Journal of Chromatography A, vol. 723, no. 2, pp. 381388, 1996.

[8] J. K. Cheng, W. H. Huang, and Z. L. Wang, "Studies on singlecell analysis," Chinese Journal of Chromatography, vol. 25, no. 1, pp. 1-10, 2007.

[9] H. F. Zou and X. D. Huang, "Separation and Determination of Biomolecules," Chinese Journal of Chromatography, vol. 21, no. 4, pp. 311-315, 2003.

[10] F. Carlucci, A. Tabucchi, A. Aiuti et al., "Capillary electrophoresis in diagnosis and monitoring of adenosine deaminase deficiency," Clinical Chemistry, vol. 49, no. 11, pp. 1830$1838,2003$.

[11] L. A. Hammad, M. M. Saleh, M. V. Novotny, and Y. Mechref, "Multiple-reaction monitoring liquid chromatography mass spectrometry for monosaccharide compositional analysis of glycoproteins ," Journal of the American Society for Mass Spectrometry, vol. 20, no. 6, pp. 1224-1234, 2009.

[12] M. Kulp, M. Vaher, and M. Kaljurand, "Miniaturization of sampling for chemical reaction monitoring by capillary electrophoresis," Journal of Chromatography A, vol. 1100, no. 1, pp. 126-129, 2005.

[13] A. Schuchert-Shi, P. Kubáň, and P. C. Hauser, "Monitoring of enzymatic reactions using conventional and on-chip capillary electrophoresis with contactless conductivity detection," Electrophoresis, vol. 28, no. 24, pp. 4690-4696, 2007.

[14] J. Wang, K. Liu, G. Sun, J. Bai, and L. Wang, "Capillary array electrophoresis for the research of asymmetric transformation reaction of L-proline to D-proline," Journal of Separation Science, vol. 28, no. 18, pp. 2551-2554, 2005.

[15] T. Yamashiro and T. Okado, "Capillary electrophoresis as a nanoreactor of separation capability: on-capillary reaction catalyzed by transition metal ions," Electrophoresis, vol. 24, no. 12-13, pp. 2168-2173, 2003.

[16] Q. Liu, L. Yao, Q. Shen, Z. Nie, M. Guo, and S. Yao, "Layer-bylayer assembly of polyelectrolyte and nanoparticles, monitored by capillary electrophoresis," Chemistry, vol. 15, no. 46, pp. 12828-12836, 2009.
[17] J. Pédron, R. Maldiney, M. Brault, and E. Miginiac, "Monitoring of hapten-protein coupling reactions by capillary zone electrophoresis: improvement of abscisic acid-bovine serum albumin coupling and determination of molar coupling ratios," Journal of Chromatography A, vol. 723, no. 2, pp. 381388, 1996.

[18] J. B. Stevenson, W. C. Hymas, and D. R. Hillyard, "A novel capillary electrophoresis-based multiplex PCR assay for detection of respiratory pathogens," Annals of Clinical \& Laboratory Science, vol. 41, no. 1, pp. 33-38, 2011.

[19] C. S. Zhang, D. Xing, and Y. Y. Li, "Applications of laser technique for polymerase chain reaction microfluic chips," Chinese Journal of Analytical Chemistry, vol. 36, pp. 259-265, 2008.

[20] A. T. Woolley, D. Hadley, P. Landre, A. J. DeMello, R. A. Mathies, and M. A. Northrup, "Functional integration of PCR amplification and capillary electrophoresis in a microfabricated DNA analysis device," Analytical Chemistry, vol. 68, no. 23, pp. 4081-4086, 1996.

[21] R. S. Sahota and M. G. Khaledi, "Nonaqueous capillary electrophoresis," Analytical Chemistry, vol. 66, no. 7, pp. 11411146, 1994. 


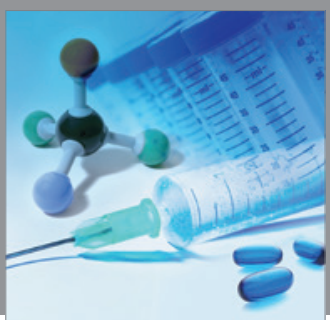

International Journal of

Medicinal Chemistry

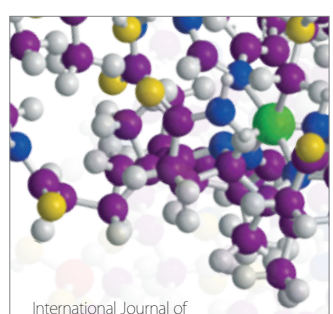

Carbohydrate Chemistry

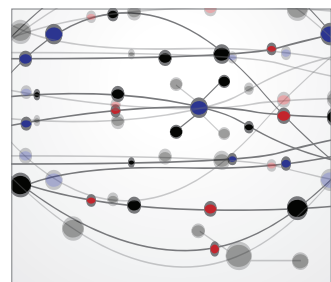

The Scientific World Journal
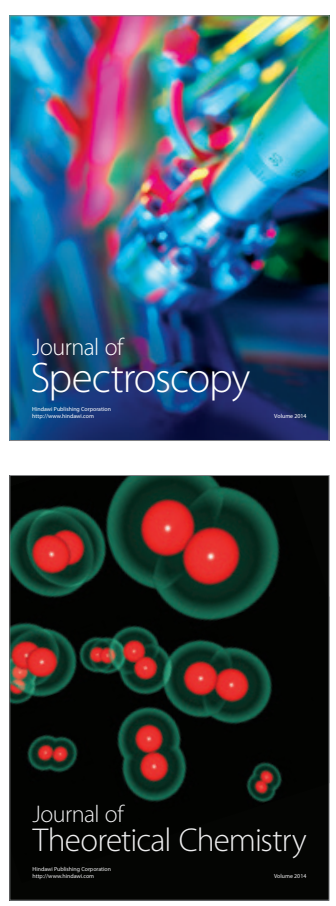
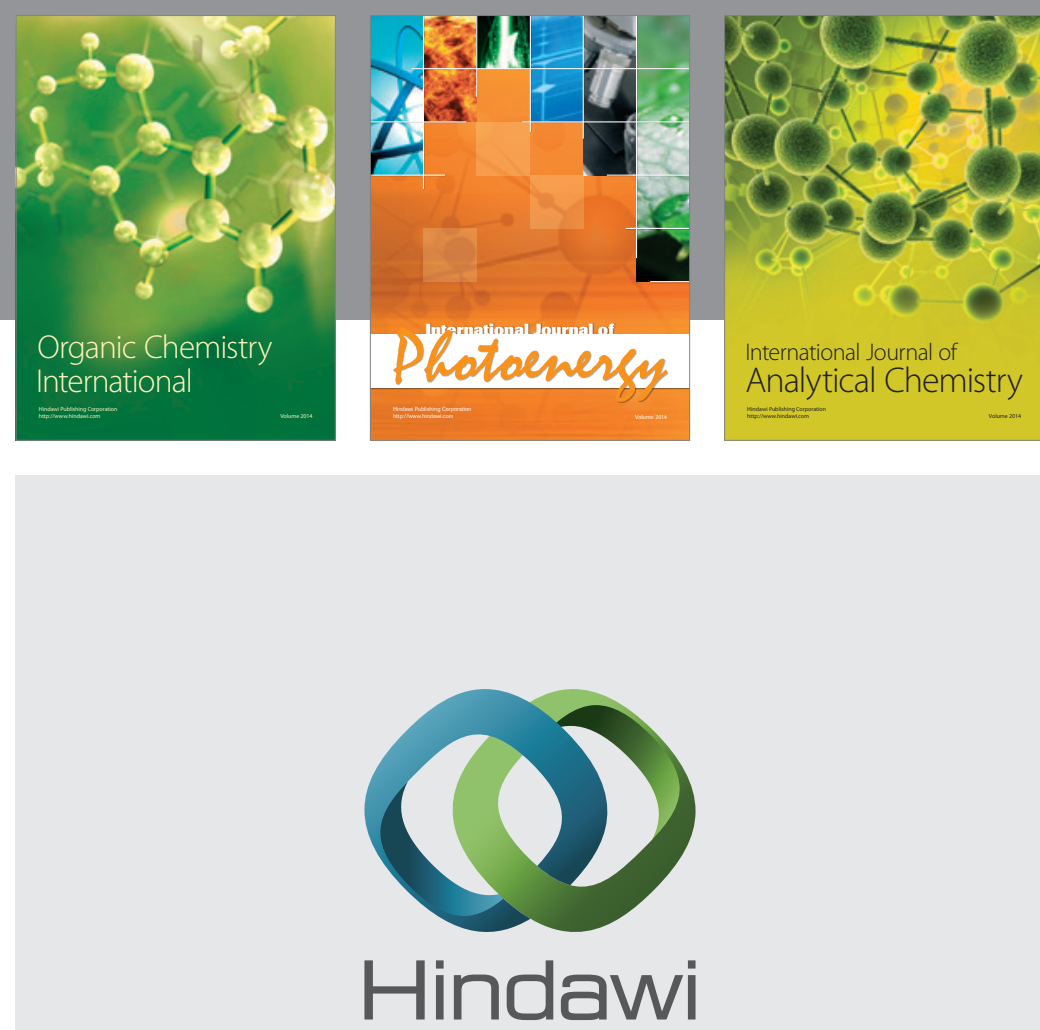

Submit your manuscripts at

http://www.hindawi.com
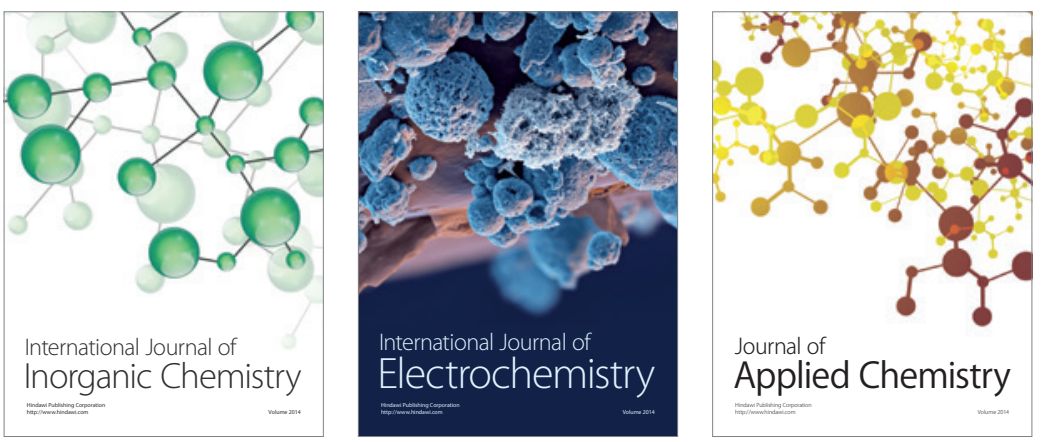

Journal of

Applied Chemistry
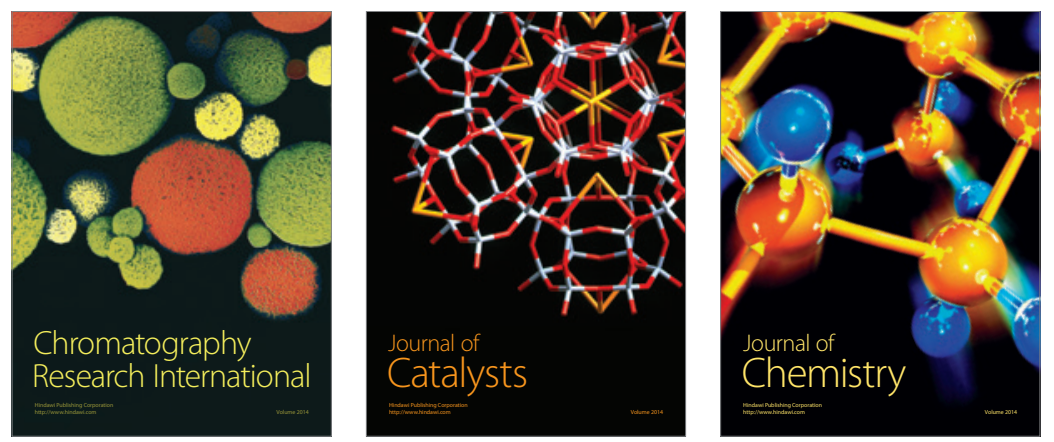
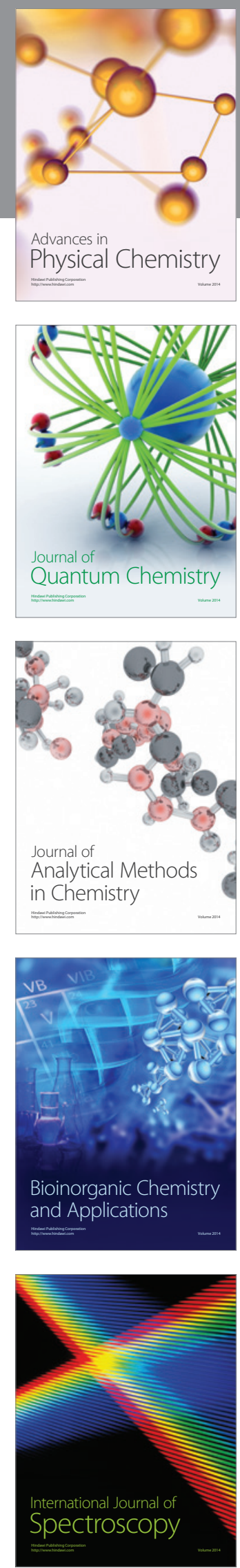